It would appear that any frequency falling between 20 and $1,000 \mathrm{c} / \mathrm{s}$ is of relevance to some clinical situation.

The stethoscopes used in this experiment were chosen as being most representative of those in general use, the exception being, of course, the wooden monaural.

An initial attempt to reproduce the experimental conditions of Rappaport and Sprague (1941) was unsuccessful, as it proved impossible to obtain an effective soundproof seal between the chest wall and a loudspeaker baffle.

Some previously enunciated principles of stethoscope design may now be examined in the light of our results.

(1) "It is apparent that the larger the area of the stethoscope piece in contact with the chest, the greater the amount of sound collected" (Macfarlan, 1938). The Leatham big bell has a performance somewhat inferior to that of the little bell; the skin area enclosed by the Sprague-Bowles bell is greater than that of the basic stethoscope, yet the performance of the Sprague-Bowles bell is inferior, though the two stethoscopes have similar tubing and binaural dimensions.

(2) A good stethoscope needs both a bell and a diaphragm chestpiece (B.M.f., 1962). The Sprague-Bowles diaphragm is superior to the bell in both the higher and lower frequencies. The Littmann diaphragm performs better than the bell in the lower frequencies; the converse applies at the higher frequencies. Both the Allen and the Leatham bell seem to be superior to their respective diaphragms at both higher and lower frequencies.

(3) A stethoscope should have a small internal volume (Barss et al., 1926 ; Rappaport and Sprague, 1951). In general our results seem to support this principle. The Leatham and the Allen both use small-bore tubing (diameter $0.3 \mathrm{~cm}$.) and have small internal volumes.

In general these results differ from those of Barss et al., (1926) and Rappaport and Sprague (1941, 1951). Barss et al. concluded that increasing the area of the chestpiece leads to greater efficiency. Their conclusions were based on an experiment involving the passage of sound from a source, through air, to the chestpiece; these conditions are very different from those obtaining in the clinical use of the stethoscope. Rappaport and Sprague compared stethoscope components rather than complete stethoscopes. The acoustic properties of any component are very different from those of a stethoscope incorporating that component.

It is suggested that in stethoscope design most of the previously accepted principles should be ignored and an empirical approach should be used. Stethoscopes of small internal volume might be modified step by step, discarding modifications leading to an inferior performance and incorporating those leading to improved performances.

The conditions of testing should correspond as closely as possible to those in clinical use.

\section{Summary}

A comparison has been made of the efficiency of various stethoscopes in common clinical use by simulating natural conditions as far as possible. Of the stethoscopes tested by this method the Leatham little bell and the Allen bell gave the best overall performances. The current prices in the U.K. of the various stethoscopes are listed. It is suggested that an empirical approach to stethoscope design is adopted, though, in general, stethoscopes with small internal volume and small endpiece skin contact gave the better performances.

We would like to thank Dr. J. H. Taylor, who originally suggested that we should attempt a Which ? report on stethoscopes, and Professor A. E. Ritchie for his help and advice. Mr. P. D. L. Constable gave invaluable help with the statistics.

\section{REFERENCES}

Barss, W. R., Eade, W. F., and Fitzgerald, E. B. (1926). Boston med. surg. 于., $195,116$.

Brit. med. $\mathscr{\text { f., }} 1962,1,856$

Dawson, J. B. (1964). Practitioner, 193, 315.

Leatham, A. (1958). Lancet, 1, 463.

Macfarlan, D. (1938). f. Amer. med. Ass., 110, 2068.

Rappaport, M E., and Sprague, H. B. (1941). Amer. Heart F., 21, 257. (1951). Ibid., 42, 605 .

\title{
Location of Placenta by Means of a Colour Autoscan Technique
}

\author{
W. THOMPSON,* M.B., B.SC., M.R.C.o.G. ; T. K. BELL, † B.SC., A.INST.P. ; J. H. M. PINKERTON,* M.D., P.R.C.O.G.
}

Brit. med.F., 1967, 4, 390-391

Location of the placenta by radioactive scanning techniques has considerable advantages over existing $x$-ray methods and is being used with increasing frequency (Hibbard, 1966). When tracer doses of isotopes tagged to human albumin are employed the radiation risk to the foetus is greatly reduced (McGee and Duron, 1960). In 1966, at the Royal Victoria Hospital, Belfast, a colour autoscan apparatus became available that could be adopted for use in placentography. This paper describes the use of this apparatus and the results so far obtained in 62 cases.

\section{Method and Equipment}

Lugol's iodine solution, $0.3 \mathrm{ml}$. three times daily for 24 hours before and seven days after the test, was given to block the maternal and foetal thyroid (Cavanagh et al., 1961).

Human serum albumin labelled with ${ }^{131} \mathrm{I}$, obtained from the Radiochemical Centre, Amersham, was diluted in saline and

- Deparment of Gynaecology, the Queen's University, Belfast. + Deparment of Medical Physics, Royal Victoria Hospital, Belfast.
5 microcuries administered intravenously to each patient. Five to 10 minutes were allowed for the radioactive compound to mix throughout the vascular department. The patient was then placed supine on the couch of a total body scanner (Isotope Developments Ltd., Reading) beneath a fixed scintillation counter in axial alignment with another similar counter below the couch. Each counter contained a 3 -in. $(7.5-\mathrm{cm}$.) diameter sodium iodide crystal in a 3-in. diameter lead collinator. The couch was mechanically moved backwards and forwards between the counters along parallel lines separated by a distance of $1 \mathrm{~cm}$. until the whole abdominal area was scanned.

The radiations detected by each counter are converted to electrical pulses, added together, and operate a striker and the six-colour ribbon beneath it. Distribution of the radioactivity is displayed on a sheet of paper in the form of coloured dashes, indicating regions of high and low concentrations. This double counter system gives higher counting rates, better distinction, and a shorter operating-time-approximately 20 minutes-as compared with the single counter placed above the patient used in earlier work in this series. 
At the end of the scan the individual counting rates from each detector viewing the "hot" region indicate whether the placenta was anterior or posterior.

\section{Results}

The indications for placentography were a suspicion of placenta praevia in 58 patients who presented with antepartum haemorrhage (52) or a persistent unstable lie at 37 weeks (6) (see Table), and the need to define the placenta before amniocentesis in four. The usual gestation period at which the test was performed was 32-33 weeks, but satisfactory results were obtained as early as 30 weeks. In four patients placenta praevia diagnosed on scanning was not confirmed clinically. These false-positive results were due mainly to overcautious assessment of the scans early in the series, and with experience it has been possible to increase the accuracy of prediction. Of 42 patients with antepartum haemorrhage 32 were allowed home for intervals of two to six weeks after placentography had indicated that the placenta was in the upper uterine segment; no foetal loss resulted.

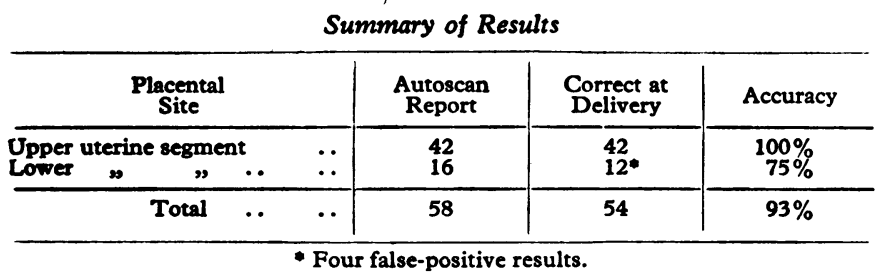

\section{Discussion}

Clinical Applications.-The accuracy of the method described is $93 \%$, and is comparable to that of other reported isotope techniques; its main clinical value is the exclusion of placenta praevia before allowing the patient home for part or all of the remainder of the antenatal period, and it is important in this connexion to note that in no patient was a placenta praevia missed. In this way we calculate that the equivalent of three out of 100 hospital beds a year are released for other purposes. No attempt was made to estimate the degree of placenta praevia, as all cases carry the risk of further possible severe haemor- rhage, and are therefore kept in hospital until after delivery. The other application of the procedure is before amniocentesis, when there is clinical evidence of an anteriorly placed placenta. Here the scan is useful in the selection of a suitable puncture site.

Choice of Isotope.-Albumin tagged with ${ }^{131}$ I was chosen as the most suitable tracer material, because it has been successfully used elsewhere for placental location (Weinberg et al., 1957 ; Heagy and Swartz, 1961 ; Durfee and Howieson, 1962), its biological characteristics are fully known, its production is reliable, it is relatively cheap, and it is readily obtained commercially. Isotopes such as ${ }^{132} I$ and technetium $99 \mathrm{~m}$ have the advantage of a much shorter half-life, so that larger quantities of radioactive material can be used, resulting in higher counting rates and better discrimination. If, however, the facilities required for routine production of albumin tagged with these isotopes are not available ${ }^{131} \mathrm{I}$ albumin has been shown to give satisfactory results.

\section{Summary}

A technique of isotope placental location is described which uses ${ }^{131} \mathrm{I}$ and a colour autoscan apparatus. The use of a twocollimator modification has been found to increase the definition of this method, so that the readily available albumin-tagged ${ }^{131} I$ gave satisfactory results.

The accuracy of the method was $93 \%$; no placenta praevia remained undetected.

We are indebted to the consultant staff of the Royal Maternity Hospital, Belfast, for permitting us to include their patients in this study. Our thanks are also due to Dr. R. Ferguson and Dr. W. B. Sproule for their help.

\section{REFERENCES}

Cavanagh, D., Powe, C. E., and Gilson, A. J. (1961). Obstet. and Gynec.,

18, 403 . 577.

Heagy, F. C., and Swartz, D. P. (1961). Radiology, 76, 936.

Hibbard, B. M. (1966). Clin Obstet. Gynec., 9, 93.

McGee, J., and Duron, D. (1960). Obstet. and Gynec., 15, 643.

Weinberg, -A., Rizzi, J., McManus, R., and Rivera, J.' (1957). Ibid., 9. 692.

\title{
Adrenocorticotrophic Action of Long-acting Tetracosactrin Compared with Corticotrophin-gel
}

\author{
G. M. BESSER,* M.D., B.SC., M.R.C.P. ; P. W. P. BUTLER,* M.B., B.S., M.R.C.P. \\ F. S. PLUMPTON, $†$ B.M., B.CH., F.F.A. R.C.S.
}

Brit. med. F., 1967, 4, 391-394

Tetracosactrin ( $\beta^{1-24}$ corticotrophin, Synacthen) is a synthetic polypeptide containing the first 24 amino-acids found in naturally occurring corticotrophin (A.C.T.H.). It has a high degree of corticotrophic activity (Landon et al., 1964) and is used to test adrenocortical responsiveness (Wood et al., 1965). However, since its action lasts only three to four hours after an intramuscular dose (Landon et al., 1964), unmodified tetracosactrin can have little place in therapy. A long-acting version of this compound might offer practical advantages over naturally occurring corticotrophin, particularly in patients who develop hypersensitivity reactions or antibodies to natural corticotrophin, since its amino-acid sequence is identical with that of

- Medical Professorial Unit, St. Bartholomew's Hospital, London E.C.1. + Department of Anaesthesia, St. Bartholomew's Hospital, London E.C.1. the active portion of human corticotrophin and the preparation does not contain any animal contamination. Tetracosactrin has now been modified by adsorption on to an inorganic zinc complex to prolong its action. This paper reports a comparison of its adrenocorticotrophic properties and length of action compared with corticotrophin-gel of animal origin in normal subjects and in patients previously treated with oral corticosteroids or corticotrophin.

\section{Methods and Subjects}

Subjects.-All subjects were volunteers without evidence of endocrine, renal, or liver disease. There were 18 non-steroidtreated subjects, of whom eight were ambulant medical staff, 